\title{
Scolia
}

Revue de linguistique

35 | 2021

Superlatifs et définitude

\section{Olga INKOVA (dir.), Autour de la reformulation}

\author{
Jean-Claude Anscombre
}

\section{OpenEdition \\ Journals}

Édition électronique

URL : https://journals.openedition.org/scolia/1558

DOI : 10.4000/scolia. 1558

ISSN : 2677-4224

\section{Éditeur}

Presses universitaires de Strasbourg

\section{Édition imprimée}

Date de publication : 9 juillet 2021

Pagination : 193-199

ISBN : 979-10-344-0091-1

ISSN : $1253-9708$

\section{Référence électronique}

Jean-Claude Anscombre, «Olga Inkova (dir.), Autour de la reformulation», Scolia [En ligne], 35 | 2021, mis en ligne le 09 juillet 2021, consulté le 13 juillet 2021. URL : http://journals.openedition.org/scolia/1558 ; DOI : https://doi.org/10.4000/scolia.1558

\section{(c) (i) (9)}

Les contenus de la revue Scolia sont mis à disposition selon les termes de la Licence Creative Commons Attribution - Pas d'Utilisation Commerciale - Partage dans les Mêmes Conditions 4.0 International. 
Olga INKOVA (dir.), Autour de la reformulation, Genève, Droz, collection Recherches et rencontres, Publication de la Faculté des Lettres de I'Université de Genève, $n^{\circ} 36,2020,216$ pages.

Cet ouvrage se compose essentiellement de deux parties. Une première partie (p. 17-117), Vers une définition, comprend cinq articles consacrés au problème d'une définition opératoire de la notion de reformulation. Une seconde partie (p. 121-190), Marqueurs et applications, étudie au travers de quatre articles divers problèmes où intervient la notion de reformulation. Complètent ce volume: une introduction de Olga Inkova (p. 7-13), une bibliographie (p. 191-212), et enfin un index des notions (p. 213-214).

Introduction (Olga Inkova): il y est rappelé brièvement le flou définitionnel qui entoure la notion elle-même de reformulation. Sorte de répétition, réinterprétation pouvant aller jusqu'à l'opposition, ou relation sémantique entre deux segments textuels: les moyens linguistiques censés réaliser la reformulation - dont des marqueurs présentent un éventail de propriétés ne prêchant pas pour une possible homogénéité de la catégorie.

Suit une présentation des différentes contributions qui composent le volume.

Coquilles et autres: Gülich \& Kotchi (1983) ne sont pas mentionnés dans les travaux consacrés aux aspects conversationnels de la reformulation, alors qu'ils déclarent explicitement vouloir « [...] en élucider les fonctions discursives et interactives ...» (p. 305). Signalons également: on pourrait notamment distinguer (p. 8 ; 1. 3); contrastifs (1. 6), difficulté (1. 8); divers cadres théoriques en vue d'une réflexion sur (p. 9, 1. 13); sans que cela soit exprimé (p.11, 1. 11).

Encore sur la notion de reformulation (Olga Inkova): la notion de reformulation, depuis ses «inventeurs» Gülich \& Kotschi (1983) jusqu'à aujourd'hui, suscite depuis plus de quarante ans l'intérêt des linguistes. Activité naturelle «innée » selon Harris, elle est centrale dans l'articulation du discours et l'interactivité discursive. C'est à un état des lieux sur ce sujet que nous convie l'auteur, en ne dissimulant pas les difficultés inhérentes à une définition un tant soit peu rigoureuse de cette notion. L'auteur nous propose sa propre classification des diverses manifestations candidates au statut de reformulation. Partant de l'idée que la reformulation est toujours de quelque manière que 
ce soit, une relation logico-sémantique entre deux «termes»- un formulé et un reformulé - Olga Inkova distingue deux paramètres qui la régissent: la nature sémantique de la relation et les moyens par lesquelles elle se manifeste. Dans le cas de la reformulation, Olga Inkova définit la reformulation comme un type de relation logico-sémantique, qu'elle explicite.

Si l'auteur fournit un parcours très clair et bien documenté parmi les méandres d'une notion aux contours souvent imprécis, les critères invoqués sont loin d'être opératoires, i.e. reproductibles, et plutôt largement intuitifs.

Coquilles, etc.: Teston-Bonnard (2015) n'apparaît pas dans la bibliographie (p. 23, 1. 4); soit au même (p. 23, 1. 11); cette définition... (p. 25, 1. 5), où est la définition?; le formulé et le reformulé restent tous les deux valides (p. 25, 1. 25); la reformulation n'a de sens (p. 28, av. dernière 1.); hyperonyme (p. 30, 1. 9, 19 et 21); élaboration (p. 31, 1. 2); Halliday (1985) ne figure pas dans la bibliographie (p. 31, 1. 7); compréhension (p. 34, n. 7); illocutoire (p. 38, 1. 30; p. 39, 1. 31) illocutif est incorrect en français; tous les niveaux (p. 39, 1. 27); en un deuxième temps (p. 40, 1. 14).

Paraphrase et reformulation: un chassé-croisé entre deux notions (Catherine Fuchs): L'auteur examine les liens existant entre paraphrase et reformulation, et plus particulièrement la problématique de la reformulation paraphrastique. Ces deux notions correspondent à deux étapes successives des études linguistiques, et à deux types de théorie. La période de la paraphrase correspond à un angle d'attaque «logique» (Harris puis la grammaire générative). Il s'agit de relations verticales: ces paraphrases relient non pas deux séquences linguistiques, mais une séquence linguistique (structure de surface) et une séquence métalinguistique (structure profonde). Dans les travaux pionniers de Gülisch, la reformulation traite des relations cette fois horizontales entre segments de discours, et en tant que pratique(s) langagière(s). Les approches contemporaines distinguent la reformulation paraphrastique et la relation non paraphrastique, la première mettant l'accent sur les marqueurs signalant la mise en équivalence des termes.

Ce texte clair et aéré constitue une intéressante mise au point sur la question. La rédaction est soignée - une seule coquille: scénarios (p. 54, 1. 10. av. la fin) - et certaines imprécisions. 
a) Les divers textes de Harris ne sont pas référencés dans la bibliographie (p. 42), non plus que Fillmore, Lakoff et Pottier (p. 43);

b) Traiter les propositions logiques en termes de valeur de vérité relève non pas de la syntaxe logique, mais de la sémantique logique (p. 45, bas de page);

c) la note 4 (p. 45), confond l'opérateur «biconditionnel» qui, à partir de deux propositions conduit à la proposition $(\mathrm{p} \rightarrow \mathrm{q}) \wedge(\mathrm{q} \rightarrow \mathrm{p})$, et la relation d'équivalence $\mathrm{p} \equiv$ q entre deux propositions: $\mathrm{p}$ est équivalent à $\mathrm{q}$ si et seulement $\mathrm{si}(\mathrm{p} \rightarrow \mathrm{q}) \wedge(\mathrm{q} \rightarrow \mathrm{p})$ est un théorème. Le reste de la note est peu clair. d) dans la note 5 (p. 46), l'exemple célébrissime l'étoile du matin/l'étoile du soir est de Frege (Sinn und Bedeutung, 1892), et non de Quine, de même que la remarque sur les contextes dits obliques.

Les reformulations des formes méta-énonciatives par excellence. Spécificités et introducteurs (Blandine Pennec): Constatant l'hétérogénéité des phénomènes regroupés sous la dénomination de reformulation, l'auteur pose la question de l'unité de la catégorie de tels phénomènes. En effet, la paraphrase renvoie à des contenus identiques; la reformulation paraphrastique se présente comme disant la même chose tout en s'éloignant du premier contenu, et la reformulation non paraphrastique enfin, dit autre chose. Pour l'auteur, l'ensemble des reformulations est à ranger parmi les phénomènes de métaénonciation: l'énonciation se prend elle-même comme objet. Les diverses reformulations peuvent alors être considérées comme des formes de réajustement, opéré au travers de marqueurs. L'étude de cas de formulations paraphrastiques et non paraphrastiques conduit l'auteur à conclure à l'unité des phénomènes de reformulation.

Rédigé de façon très claire, ce texte a le mérite de chercher l'unité de fonctionnement derrière la multiplicité des réalisations, en termes de réajustements opérant à des niveaux différents.

Coquilles: Rossari (p. 63, note 6); interlocutif (p. 65, 1. 24) n'existe pas en français, on doit dire interlocutoire.

Peut-on aborder la notion de reformulation autrement que par la typologie des marqueurs? (Hélène Vassiliadou): Hélène Vassiliadou explore et compare deux voies d'accès à la notion de reformulation: une définition stricte du mot (et donc de l'objet) reformulation; et l'hypothèse selon laquelle existeraient des marqueurs prototypiques de reformulation. Après avoir examiné les différentes options possibles, 
l'auteur propose de définir reformuler comme signifiant «[...] formuler une deuxième fois dans le but d'éclaircir le sens d'un segment antérieur $[\ldots]$ ». La seconde hypothèse conduit l'auteur à examiner la pertinence de la présence/absence de marqueurs pour tester la présence d'une possible reformulation. L'auteur choisit dans ce travail l'étude de l'insertion de marqueurs comme possible critère, tout en remarquant le caractère ouvert et hétérogène des listes de marqueurs proposés, ainsi que la polyvalence de la plupart des marqueurs en général.

Cet exposé a le mérite de pointer du doigt les problèmes qui se présentent quand on aborde la réalisation effective ou non d'une reformulation, mais ne fournit pas de définition de la notion de base.

Quelques coquilles: Schiffrin (1987), p. 86; Mortureux (1982), p. 91, Hansen (1996), p. 92, sont cités dans le texte et non dans la bibliographie. Il existe des études sur la syntaxe et la prosodie des marqueurs antérieures à Vassiliadou (2020b). Corollairement (p. 78, 5 1. av. la fin); les solutions attendues de leur création (p. 79, n. 3); qui ne va pas de soi (p. 80, 1.26); a fait une véritable erreur de syntaxe ou un contresens (p. 84, 8 l. av. la fin).

Peut-on parler d'une reformulation interdiscursive? (Houda Landolsi): Sur la base d'un discours politique spécifique (corpus oral), Houda Landolsi s'interroge sur le possible statut de reformulation du déjà-dit, phénomène courant dans de tels discours. Elle définit la reformulation comme le retour (énoncé reformulé) sur une première formulation (énoncé source) afin d'en modifier un des aspects. Dans toute reformulation figure un invariant, d'ordre sémantique ou argumentatif. La reformulation paraphrastique a ainsi comme invariant une équivalence sémantique. Enfin, toute reformulation ne requiert pas nécessairement la présence d'un marqueur. La conception de la reformulation se situe donc entre le rephrasage (même forme pour la source et le reformulé) et la réinterprétation (anti-orientation argumentative de deux segments). Houda Landolsi complète sa conception de la reformulation par celle d'interdiscours, défini comme "l'effet d'un discours sur un autre (p. 103)», et dont la perspective rejoint celle de l'hétérogénéité discursive chère à J. Authier-Revuz. Elle crée ainsi le concept de reformulation interdiscursive, processus de reprise explicite ou implicite d'une formulation appartenant à un discours autre. 
La principale qualité de ce texte est l'effort que fait son auteur pour définir de façon explicite les différents concepts forgés pour introduire un type de reformulation original.

Le texte est très soigné, mais cependant Rabatel (2010), p. 105, 7 lignes av. la fin, ainsi que Lochak (2007), p. 114, 1. 2, sont absents de la bibliographie. Ce discours a été provenu (p. 106, 1. 25) est impossible en français. Page 100: on aurait aimé quelques précisions sur la nature de l'invariant dans le cas de la reformulation non paraphrastique. Page 111, l. 14 sq. : il n'y a pas dans Ducrot (1984), d'introduction en polyphonie d'une notion théorique de point de vue. Une telle notion apparaît chez Nølke (1993) - et ne peut donc être attribuée à Rabatel. Par ailleurs, la conception du point de vue en polyphonie n'est pas celle d'un contenu propositionnel susceptible de valeurs de vérité.

BREF (Alain Berrendonner): S'intéressant au morphème bref, Berrendonner s'attaque à deux qualifications souvent émises à son propos, à savoir qu'il s'agit: a) syntaxiquement, d'un adverbe d'énonciation, lesquels sont une sous-espèce particulière des adverbes de phrase; b) d'un point de vue sémantico-pragmatique, d'un connecteur reliant la phrase à laquelle il s'adjoint à une ou plusieurs phrases antérieures, cette même phrase constituant une récapitulation /reformulation de ces phrases antérieures. Procédant à l'étude détaillée de la prosodie de bref - et aussi de sa syntaxe, Berrendonner en conclut que: a) bref exhibe les contours prosodiques d'un énoncé isolé; b) dans les suites textuelles de type bref $+\mathrm{P}$, rien n'indique l'existence d'une relation de dépendance syntaxique entre bref et $\mathrm{P}$. D'où la conclusion que bref forme à lui seul un énoncé distinct, grammaticalement autonome. Étudiant ensuite le statut de reformulation de bref, Berrendonner, au travers d'une série d'exemples détaillés, montre que le seul invariant commun est une instruction de clôture d'une unité discursive dont bref signale l'abandon par le locuteur. Bref n'est donc qu'un simple commentaire portant sur ce qui le précède, il peut donc se faire qu'il soit compatible avec diverses interprétations, dont la reformulation.

Coquilles: Tesnières (1969) et Kallen-Tatarova (2013) sont cités dans le texte, mais pas dans la bibliographie.

De AINZ à PLUTÔT. Un cycle de grammaticalisation (Maj-Britt Mosegaard-Hansen): Cette étude montre que les deux connecteurs ainz (forme longue ainçois), obsolète dès le français classique et plus tôt 
(forme lexicalisée plutôt), bien que distants dans le temps, ont connu des évolutions sémantico-pragmatiques similaires suivant le schéma adverbe temporel comparatif $>$ marqueur pragmatique exprimant une préférence $>$ marqueur de reformulation non paraphrastique. Après une étude approfondie tant diachronique que synchronique des deux marqueurs et de leur(s) fonctionnement(s), l'auteur en conclut que les deux marqueurs ont connu des évolutions très semblables, relevant d'un processus de pragmaticalisation. Tant ainz que plutôt semblent avoir parcouru des cycles de pragmaticalisation, passant d'usages vériconditionnels à des usages pragmatiques, l'usage vériconditionnel étant alors occupé par un autre marqueur qui développe à son tour des usages pragmatiques. Il semble qu'existent d'autres cas semblables: l'hypothèse de tels cycles fournirait ainsi un instrument de reconstruction interne du sémantisme des langues.

Coquilles: Taavitsaineen\& Jucker (2008) est cité dans le texte et n'apparaît pas dans la bibliographie.

La notion de reformulation dans le domaine du TAL (Iris EshkolTaravella \& Natalia Grabar): Ce travail est une présentation de la façon dont la notion de reformulation est intégrée dans le Traitement Automatique des Langues (TAL). Dans les travaux du TAL, le repérage automatique de reformulations fait partie de tâches comme la détection de plagiats, l'inférence textuelle, la traduction automatique, etc. La reformulation peut être vue comme une notion paradigmatique pour l'étude de corpus écrits, proche dans son principe de la paraphrase, approche à la base de la théorie Sens-Texte de Mel'čuk. Elle peut être également vue, dans les travaux sur des données orales, selon une approche syntagmatique de disfluence. Ce travail présente une synthèse de travaux menés sur l'analyse outillée et le traitement automatique de reformulations dans des corpus non normalisés (oral transcrit et forums du Web); un exemple concret est présenté et analysé.

Coquilles: Rossari (1993), Dagan et alii (2013), Madnani \& Dorr (2010), Mel'čuk et alii (1984, 2000), Malakasiotis \& Androutsopoilos (2007), Dagan et alii (2005, 2013), sont cités dans le texte et n'apparaissent pas dans la bibliographie.

Les phénomènes de réitération dans les interactions logopédisteadolescent (Audrey Sublon et Geneviève de Weck) : Étude de différents phénomènes de réitération, dans une approche socio-interactionnelle de l'acquisition du langage et des troubles qui lui sont associés. L'approche 
méthodologique a été adaptée à des interactions asymétriques impliquant des adolescents. En définissant la relation formelle de la réitération à la source, on peut en distinguer quatre types: reprise de forme à l'identique (répétition), modification formelle (reformulation), reproduction d'une construction syntaxique (réitération de structure), et enfin combinaisons de plusieurs types (compilation). Les différences d'utilisation des réitérations entre les participants montrent une influence de de l'asymétrie générale de l'interaction et des rôles respectifs à l'intérieur de celle-ci.

Coquilles: Bernicot et alii (2006) - deux références dans la bibliographie.

Ce volume intéressera tous ceux qui désirent s'informer sur l'état de la question de la reformulation. Les articles sont rédigés de façon claire et documentée, certains sont très stimulants, notamment ceux de Houda Landolsi, Alain Berrendonner et Maj-Britte MosegaardHansen. On peut regretter certaines erreurs et coquilles, le fait que beaucoup d'articles cités dans les textes n'apparaissent pas dans la bibliographie et qu'inversement, un nombre important de textes cités dans la bibliographie (environ 20\%) ne soient pas appelés dans les textes. Le plus gros reproche qu'on peut cependant adresser à ce collectif est qu'après l'avoir lu, on ne sait toujours pas ce qu'est la reformulation, i.e. cette notion n'est pas caractérisée par des propriétés linguistiques objectivement repérables.

Jean-Claude ANSCOMBRE

Directeur de Recherche émérite

Centre Jean Pruvost (CNRS-LT2D, EA 7518)

Université de Cergy-Pontoise

jean-claude.anscombre@orange.fr

\section{Références}

GÜLICH E. \& KOTSCHI T. (1983), Les marqueurs de la reformulation paraphrastique, Cahiers de linguistique française 5, 305-351. 